\title{
OBSERVAÇÕES SOBRE A POLINIZAÇÃO DE MELÃO (Cucumis melo L.) CULTIVADO EM ESTUFA
}

Luís Carlos Marchini ${ }^{1}$, Daniela de Almeida ${ }^{2}$, M. A. N. Vásquez ${ }^{2}$, Augusta C.de C.C. Moreti ${ }^{3}$, Ivani Pozar Otsuk ${ }^{3}$

\section{RESUMO}

Este trabalho, sobre o comportamento de abelhas na polinização de melões em áreas cobertas, foi conduzido no campo experimental do Departamento de Engenharia Rural da Escola Superior de Agricultura Luiz de Queiroz/USP, em Piracicaba, SP, numa área protegida de $396,20 \mathrm{~m}^{2}$. Utilizaram-se sementes do híbrido de melão Bônus No.2 do tipo rendilhado, com duas fileiras de plantas, espaçadas de $0,6 \mathrm{~m}$ entre plantas e $0,20 \mathrm{~m}$ entre fileiras, em esquema alternado. Inicialmente realizou-se polinização manual em cerca de 30 botões florais, devidamente marcados; posteriormente, introduziu-se no ambiente um núcleo de abelhas Apis mellifera, responsável pela polinização das demais flores. Os parâmetros determinados para os frutos colhidos foram: peso $(\mathrm{g})$; diâmetro $(\mathrm{cm})$; comprimento (cm); graus Brix $\left(^{\circ}\right)$; acidez (meq $\mathrm{kg}^{-1}$ ) e pH. Foram analisados 26 melões que receberam polinização manual e 35 resultantes da polinização feita pelas abelhas. Verificou-se maior eficiência da polinização nelitófila, resultando em melões com melhores características e ausência de frutos deformados.

Palavras-chave: abelhas, polinização, aumento de produção, melão

Professor - Escola Superior de Agricultura "Luiz de Queiroz"/USP, Piracicaba, SP, 3rasil.

! Mestrando - Escola Superior de Agricultura "Luiz de Queiroz"/USP, Piracicaba, ;P, Brasil.

Pesquisadora- Instituto de Zootecnia/APTA/SAA, Nova Odessa, SP, Brasil 


\section{CANTALOUPE (Cucumis melo L.) POLLINATION IN COVERED}

\section{AREAS}

\section{ABSTRACT}

This paper deals with the influence of honey bees pollination on cantaloupes in covered areas. The experiment was set in a protected area $\left(396.20 \mathrm{~m}^{2}\right)$ of the Department of "Engenharia Rural, Escola Superior de Agricultura Luiz de Queiroz (ESALQ)", University of São Paulo, in Piracicaba, State of SãoPaulo, Brazil. Seeds of the cantaloupeshybrid Bond No. 2 of the "rendilhado" type were sowed, in two rows of plants, $0.60 \mathrm{~m}$ between plants and $0.20 \mathrm{~m}$ between rows. Firstly the pollination was manually done on 30 floral buttons, later on one introduced a nucleus of honey bees Apis mellifera, responsible for pollinating the rest of flowers in the protected area. The parameters determined for the harvested fruits were: weight $(\mathrm{g})$; diameter $(\mathrm{cm})$; length $(\mathrm{cm})$; $\operatorname{Brix}\left({ }^{\circ}\right)$; acidity $\left(\mathrm{meq} \mathrm{kg}^{-1}\right)$ and $\mathrm{pH}$. A total of 26 cantaloupes that had received hand pollination and 35 which received bee pollination were analyzed. One observed that the bee pollination was most efficient, resulting in cantaloupes with better characteristics the absence of deformed fruits.

Key words: honeybees, pollination, increasing production, cantaloupe

\section{INTRODUÇÃO}

A polinização é a transferência do pólen da antera (órgão masculino) para o estigma, órgão feminino da planta, propiciando a germinação do pólen, ocasionando a produção de frutos e sementes. Observa-se, em muitas espécies vegetais, uma dependência íntima com os agentes polinizadores para sua continuidade como espécie. Para aumentar a atração dos agentes, muitas plantas, além de produzirem flores coloridas, perfumadas e com néctar rico em açúcares, se especializaram a ponto de 
apresentarem coloração, formato e disposição de pêlos que imitam o corpo do agente polinizador $\grave{A}$ medida que a agricultura vai se desenvolvendo, com o aprimoramento das técnicas de cultivo (como o plantio em áreas fechadas), de fertilização e irrigação do solo, de utilização de sementes selecionadas, a polinização pode se tornar um fator limitante na produção agrícola dos países tropicais. Dentre os agentes polinizadores, a abelha Apis mellifera possui um papel de destaque devido à visita de um grande número de flores de plantas de uma mesma espécie, por carregarem em seus pêlos ramificados numerosos grãos de pólen, por ocorrerem em grande número na natureza e terem a capacidade de coletar em grandes quantidades (Couto e Couto, 1996).

São inúmeros os parâmetros que influenciam o número de colméias por unidade de área, usado para a polinização de uma cultura, como por exemplo: clima, espécies e números de abelhas, técnicas de cultivo e insetos silvestres existentes. Segundo Free (1993), quando a cultura é atrativa, mas suas flores são comparativamente esparsas ou podem ser autopolinizadas, são necessárias menos que 2,5 colônias/ha, para uma eficiente polinização. Insetos polinizadores de culturas aumentam não somente a quantidade, mas também melhoram a qualidade do fruto, como ocorre em Cucumis melo. McGregor e Todd (1952) foram os primeiros autores a demonstrar a importância das abelhas na polinização dos melões quando em culturas abertas contendo abelhas, obtiveram 145 melões e nas plantas sem abelhas, apenas 4 . Observaram também que os melões oriundos de flores mais novas são mais doces e mais desenvolvidos do que das flores produzidas mais tardiamente. Os dados foram confirmados por Alex (1957) que obteve em média 3,9 melões/planta protegida contendo abelhas e 0,4 para plantas sem abelhas.

Taylor (1955) comparou a produção de frutos de 17 campos sem abelhas com a de 20 campos contendo colônias de abelhas (média de 1 
colônia/0,8ha), observando uma produção de 0,7 e 1,1 melão/planta respectivamente. Segundo D'Aguilar et al. (1967), quando uma colônia de abelhas foi colocada no final de uma casa de vegetação contendo melão, poucas abelhas forragearam as plantas mais distantes da colméia; assim, para ter uma mesma distribuição de abelhas em ambiente protegido, o indicado seria colocar uma colméia no centro da área. Iselin et al. (1974), observando a polinização de melão (Cucumis melo) por abelhas em estufa inflável, verificaram que as flores isoladas das abelhas produziram frutos não comercializáveis. Também verificaram que a produção de plantas com flores expostas às abelhas por diferentes períodos, não variou significativamente e que as abelhas alimentadas com suplemento de pólen, tiveram sua atividade aumentada.

Em casas de vegetação do Japão, a polinização por abelhas foi mais eficiente que a manual, produzindo frutos em $98 \%$ das flores (Sakamori et al., 1977). Na Itália, em estufas plásticas sem abelhas praticamente não se produziu melão (Celli e Giordani, 1981), na Bélgica Lemasson (1987) comparou estufas com e sem abelhas, verificando aumento na produção de frutos de $18,5 \%$ em comparação com os $2,5 \%$ produzidos sem visita de abelhas, com peso de frutos de $621 \mathrm{~g}$ em comparação com os $491 \mathrm{~g}$ sem visita de abelhas e número de frutos por planta de 4,3 em média em comparação com os 2,9 sem visita de abelhas. Williams (1987), na Austrália, observou que em áreas cobertas para excluir abelhas, áreas cobertas contendo abelhas no seu interior e áreas descobertas produziram, respectivamente, 20, 26 e 27 melões, com média de peso de $0,68,1,11$ e $1,10 \mathrm{~kg}$ cada. Holanda Neto et al. (2000), estudando a atratividade de flores de diferentes híbridos de melão, no Estado do Ceará, Brasil, verificaram que existem diferenças de atratividade entre eles sendo os mais atrativos Matisse, Hymark e Mission que receberam 146,75 \pm 11,$00 ; 151,25 \pm 9,56$ e 156,00 $\pm 8,67$ indivíduos por dia, respectivamente. 
Fávero et al. (2002), também no Brasil, verificaram que os insetos mais freqüentes em melões produzidos por hidroponia, em foram as abelhas da espécie Apis mellifera L. (86\% do total) e coleópteros (14\%). O horário de maior visitação das abelhas foi das 09 às 12 horas. Kato e Couto (2002) estudando, no Brasil, a polinização entomófila do melão (cultivar tipo amarelo) no Nordeste (campo aberto) e no sul (tipo rendilhado), verificaram que em ambos os cultivares há necessidade de agentes polinizadores externos.

\section{MATERIAL E MÉTODOS}

O experimento foi conduzido no campo experimental do Departamento de Engenharia Rural da Escola Superior de Agricultura Luiz de Queiroz - USP, Piracicaba-SP, em uma área protegida de $396.20 \mathrm{~m}^{2}$. O solo da área de plantio foi classificado como terra Roxa Estruturada (Alfisol) série Luiz de Queiroz, textura moderadamente argilosa, baixo conteúdo de M.O. e pH de 4,5. Foram incorporados 7,12 t.ha ${ }^{-1}$ de calcário dolomítico, 60 dias antes do transplantio das mudas, elevando a saturação por bases para $80 \%$ (IAC, 1996); a adubação de fundação foi feita por cova com $12 \mathrm{~kg}$ de esterco bovino e $0,052 \mathrm{~kg}$ de $\mathrm{P}_{2} \mathrm{O}_{5}$ (termofosfatado com micronutrientes). Utilizaram-se sementes do híbrido de melão Bônus No. 2 do tipo rendilhado, com duas fileiras de plantas, espaçados de $0,60 \mathrm{~m}$ entre plantas e $0,20 \mathrm{~m}$ entre fileiras, em esquema alternado. A fertirrigação foi feita à base de $150 \mathrm{~kg} \cdot \mathrm{ha}^{-1}$ de nitrogênio para cada tratamentos, as fontes foram nitrato de amônia ( $45 \%$ de N) e nitrato de potássio (33\% de N e 45\% de $\mathrm{K}_{2} \mathrm{O}$ ), segundo Bar-Yosef (1999), com freqüência de dois dias.

O sistema de irrigação foi gotejamento, auxiliado por um demicrocontrolador Basic Step e um periférico, previsto de válvulas tipo solenóide, possibilitando o fluxo a rede hidráulica de 24 trechos de polietileno com $13 \mathrm{~mm}$ de diâmetro dominal, comprimento entre $24 \mathrm{~m}$ a 64 
$m$ e pressão nominal de 15 mca. Foram utilizados gotejadores autocompensantes modelo RAM 17, espaçados de $0,30 \mathrm{~m}$ e dispostos no centro das duas fileiras de plantas. Os tratos culturais foram executados sempre que se fizeram necessários, evitando-se qualquer interferência externa no experimento. A polinização foi inicialmente realizada de forma manual em cerca de 30 botões florais, devidamente marcados, e posteriormente, foi acrescido no centro do ambiente um núcleo de abelhas Apis mellifera, responsáveis pela polinização das demais flores. Os parâmetros analisados dos frutos colhidos, quando maduros, foram: peso (g); diâmetro $(\mathrm{cm})$; comprimento $(\mathrm{cm})$; graus Brix $\left({ }^{\circ}\right)$; acidez (meq/kg) e pH. Foram analisados 26 melões que receberam polinização manual e 35 resultantes da polinização feita pelas abelhas.

O delineamento experimental utilizado foi o inteiramente ao acaso com dois tratamentos: polinização manual e por abelhas, com 26 repetições para o primeiro e 35 para o segundo. Os dados obtidos para peso foram transformados em $\log \mathrm{x} e$ os referentes à Brix, foram transformados em log 10 para a análise.

\section{RESULTADOS E DISCUSSÃO}

Os resultados obtidos quanto ao peso, diâmetro, comprimento, Brix, acidez e pH dos frutos de melão obtidos por polinização manual encontram-se na Tabela 1 e os obtidos para frutos produzidos por polinização com abelhas Apis mellifera encontram-se na Tabela 2. Os frutos polinizados por abelhas apresentaram peso e diâmetro significativamente maiores do que os polinizados manualmente (Tabela 3). Os resultados referentes a Brix, acidez, $\mathrm{pH}$ e comprimento, no entanto, não diferiram entre os frutos polinizados por abelhas ou manualmente (Tabela 3). 
Tabela 1. Peso, diâmetro, comprimento, Brix, acidez e $\mathrm{pH}$ de frutos de melão que tiveram polinização manual

\begin{tabular}{|c|c|c|c|c|c|c|}
\hline Repetição & Peso (g) & $\begin{array}{l}\text { Diâmetro } \\
(\mathrm{cm})\end{array}$ & $\begin{array}{l}\text { Comprim } \\
\text { ento }(\mathrm{cm})\end{array}$ & $\operatorname{Brix}\left({ }^{\circ}\right)$ & $\begin{array}{c}\text { Acidez } \\
(\mathrm{meq} / \mathrm{kg})\end{array}$ & $\mathrm{pH}$ \\
\hline 1 & 534,5 & 100,77 & 99,93 & 9,0 & 0,077 & 5,90 \\
\hline 2 & 832,5 & 111,60 & 120,06 & 10,0 & 0,096 & 5,97 \\
\hline 3 & 522,5 & 98,72 & 99,43 & 7,5 & 0,096 & 6,12 \\
\hline 4 & 554,5 & 100,33 & 100,20 & 7,5 & 0,096 & 6,12 \\
\hline 5 & 372,5 & 87,12 & 88,48 & 9,2 & 0,160 & 6,01 \\
\hline 6 & 776,5 & 110,99 & 108,84 & 8,0 & 0,096 & 5,83 \\
\hline 7 & 445,5 & 96,85 & 92,20 & 9,0 & 0,096 & 6,79 \\
\hline 8 & 928,5 & 122,47 & 117,98 & 10,5 & 0,134 & 5,79 \\
\hline 9 & 887,0 & 116,62 & 124,92 & 10,5 & 0,115 & 5,92 \\
\hline 10 & 095,0 & 119,71 & 117,24 & 9,0 & 0,083 & 5,98 \\
\hline 11 & 711,5 & 109,90 & 110,99 & 8,0 & 0,077 & 6,35 \\
\hline 12 & 723,0 & 110,00 & 108,23 & 14,0 & 0,115 & 6,46 \\
\hline 13 & 701,0 & 106,11 & 115,82 & 7,0 & 0,090 & 6,01 \\
\hline 14 & 675,5 & 108,19 & 110,64 & 7,0 & 0,064 & 6,20 \\
\hline 15 & 692,5 & 107,27 & 113,87 & 12,0 & 0,096 & 6,40 \\
\hline 16 & 571,0 & 101,63 & 104,15 & 9,0 & 0,058 & 6,90 \\
\hline 17 & 799,0 & 109,45 & 118,98 & 8,0 & 0,090 & 6,01 \\
\hline 18 & 955,0 & 117,25 & 127,52 & 6,5 & 0,077 & 6,32 \\
\hline 19 & 703,5 & 107,26 & 116,54 & 12,0 & 0,083 & 6,39 \\
\hline 20 & 649,0 & 104,53 & 110,17 & 8,5 & 0,096 & 6,28 \\
\hline 21 & 857,0 & 113,65 & 120,33 & 9,5 & 0,058 & 6,51 \\
\hline 22 & 829,5 & 116,08 & 116,78 & 6,0 & 0,058 & 6,51 \\
\hline 23 & 690,5 & 109,93 & 104,95 & 8,0 & 0,083 & 6,11 \\
\hline 24 & 619,0 & 105,32 & 102,79 & 8,0 & 0,096 & 5,98 \\
\hline
\end{tabular}




\begin{tabular}{lrrrrrr}
25 & 746,5 & 110,74 & 113,81 & 7,0 & 0,090 & 5,90 \\
26 & 585,5 & 102,32 & 101,45 & 11,0 & 0,141 & 6,17 \\
\hline Valores & 955,5 & 122,47 & 127,52 & 14,0 & 0,160 & 6,90 \\
máximos & 372,5 & 87,12 & 88,48 & 6,0 & 0,058 & 5,79 \\
e mínimos & & & & & \\
\hline
\end{tabular}

Tabela 2. Peso, diâmetro, comprimento, Brix, acidez e $\mathrm{pH}$ de frutos de melão que tiveram polinização com abelhas

\begin{tabular}{lcccccc}
\hline Repetição & Peso $(\mathrm{g})$ & $\begin{array}{c}\text { Diâmetro } \\
(\mathrm{cm})\end{array}$ & $\begin{array}{c}\text { Comprim } \\
\text { ento(cm) }\end{array}$ & Brix $\left(^{\circ}\right)$ & $\begin{array}{c}\text { Acidez } \\
(\mathrm{meq} / \mathrm{kg})\end{array}$ & $\mathrm{pH}$ \\
\hline 1 & 885,0 & 114,45 & 116,96 & 11,0 & 0,115 & 6,14 \\
2 & 675,5 & 107,4 & 102,93 & 13,0 & 0,109 & 6,35 \\
3 & 809,0 & 114,59 & 111,12 & 12,0 & 0,128 & 6,13 \\
4 & 638,5 & 105,6 & 107,60 & 7,5 & 0,096 & 6,12 \\
5 & 560,0 & 100,18 & 105,78 & 7,5 & 0,096 & 6,12 \\
6 & 817,5 & 113,28 & 116,32 & 9,2 & 0,160 & 6,01 \\
7 & 538,0 & 101,16 & 100,16 & 10,2 & 0,160 & 6,01 \\
8 & 1076,0 & 120,3 & 131,92 & 12,0 & 0,122 & 6,31 \\
9 & 829,0 & 115,02 & 111,69 & 8,0 & 0,115 & 5,78 \\
10 & 508,5 & 97,24 & 95,81 & 7,0 & 0,096 & 5,85 \\
11 & 502,5 & 99,77 & 91,11 & 10,0 & 0,102 & 6,35 \\
12 & 862,5 & 119,52 & 115,75 & 11,0 & 0,122 & 6,46 \\
13 & 563,0 & 100,57 & 104,29 & 30,0 & 0,080 & 4,90 \\
14 & 725,5 & 112,30 & 111,50 & 7,0 & 0,064 & 6,33 \\
15 & 813,0 & 112,36 & 119,03 & 6,5 & 0,051 & 6,31 \\
16 & 704,5 & 110,04 & 110,09 & 9,0 & 0,090 & 6,17 \\
17 & 670,0 & 107,89 & 109,52 & 11,0 & 0,077 & 6,52 \\
18 & 776,0 & 111,75 & 113,26 & 14,0 & 0,134 & 6,30
\end{tabular}


19

20

21

22

23

24

25

26

27

28

29

30

31

32

33

34

35

$$
737,5
$$

108,48

119,39

12,0

0,115

6,17

686,0

107,28

109,74

13,0

0,109

6,29

878,5

$114,70 \quad 124,23$

8,0

0,083

6,21

677,1

109,26

106,74

8,0

0,05

6,52

811,0

118,11

113,24

10,0

0,102

5,88

643,5

104,84

107,44

12,0

0,11

6,25

700,5

107,64

112,63

8,0

$0,070 \quad 6,08$

1106,5

126,55

128,76

8,0

0,064

6,35

113,34

113,21

9,0

0,070

6,58

$120,10 \quad 118,57$

9,0

$0,070 \quad 6,60$

1058,5

120,89

134,41

8,5

0,102

6,05

929,0

$118,64 \quad 126,97$

9,0

0,058

6,51

1093,0

125,67

129,94

12,0

$0,115 \quad 6,68$

1070,0

141,46

152,07

9,0

$0,083 \quad 6,60$

793,5

114,34

109,35

9,0

$0,122 \quad 6,01$

$721,5 \quad 108,48 \quad 112,20$

11,0

0,115

6,10

929,0

118,64

7,5

$0,058 \quad 6,51$

\begin{tabular}{lrrrrrr}
\hline Valores & 1106,50 & 141,46 & 152,07 & 30,0 & 0,160 & 6,68 \\
máximos & 502,50 & 92,24 & 91,11 & 6,5 & 0,051 & 4,90 \\
e mínimos & & & & & & \\
\hline
\end{tabular}


Tabela 3. Valores médios de peso, diâmetro, comprimento, Brix, acidez e $\mathrm{pH}$ de frutos de melão que tiveram polinização manual e por abelhas

\begin{tabular}{lcccccc}
\hline Tratamento & Peso $(\mathrm{g})$ & $\begin{array}{c}\text { Diâmetro Comprime } \\
(\mathrm{cm})\end{array}$ & Bto $(\mathrm{cm})$ & & $\begin{array}{c}\text { Acidez } \\
(\mathrm{meq} / \mathrm{kg})\end{array}$ & $\mathrm{pH}$ \\
\hline $\begin{array}{l}\text { Plantas } \\
\text { polinizadas }\end{array}$ & 702,6 & $107,88 \mathrm{~b}$ & $110,24 \mathrm{a}$ & 8,91 & $0,094 \mathrm{a}$ & $6,18 \mathrm{a}$ \\
manualmente & $(2,79 \mathrm{~b})$ & & & $(0,94 \mathrm{a})$ & & \\
$\begin{array}{l}\text { Plantas } \\
\text { polinizadas }\end{array}$ & 782,58 & $116,08 \mathrm{a}$ & $114,13 \mathrm{a}$ & 9,43 & $0,101 \mathrm{a}$ & $6,20 \mathrm{a}$ \\
por abelhas & $(2,88 \mathrm{a})$ & & & & & \\
\hline CV\% & & & & $(0,99 \mathrm{a})$ & & \\
$\mathrm{P}$ & 5,08 & 7,58 & 9,72 & 11,25 & 27,74 & 4,97 \\
& 0,0236 & 0,0328 & 0,1080 & 0,0825 & 0,4911 & 0,7448 \\
\hline
\end{tabular}

Médias seguidas de diferentes letras nas colunas diferem significativamente pelo teste $\mathrm{F}(\mathrm{P}<0,05)$

Comparando o peso de frutos com os obtidos por Garcia et al. (2005), verifica-se que tanto os melões polinizados manualmente no presente trabalho (média de $702,6 \mathrm{~g}$ - Tabela 3 ) quanto os polinizados por abelhas $(782,58 \mathrm{~g}$ - Tabela 3$)$ foram superiores ao observados pelos autores $(622,38 \mathrm{~g}$ e $711,51 \mathrm{~g}$, respectivamente), embora em ambos os trabalhos a diferença de peso tenha sido significativa em favor dos frutos polinizados por abelhas. Estes autores ainda verificaram que o diâmetro dos frutos de plantas polinizadas por abelhas foi superior ao das plantas polinizadas manualmente, da mesma forma que ocorreu no presente estudo.

Observações complementares permitem afirmar que no presente trabalho, a área polinizada por abelhas não apresentou frutos deformados, fato normalmente constatado em culturas sem a presença do inseto polinizador. 


\section{CONCLUSÃO}

A polinização realizada por abelhas (Apis mellifera) na cultura do melão é vantajosa em comparação à polinização manual, pois além de propiciar frutos com peso e diâmetro maiores, dispensa mão-de-obra trabalhosa, evitando a possibilidade de aparecimento de frutos deformados.

\section{REFERÊNCIAS BIBLIOGRÁFICAS}

ALEX, A.H. Honeybees aid pollination of cucumbers and cantaloupes. Glean. Bee Cult., v. 85, p. 398-400, 1957.

BAR-YOSEF, B. Advances in fertilization. In: Advances in Agronomy, New York. 1999, v. 65, p.1-77.

CELLI, G., GIORDANI, G. Importanza economica e biologica dell'ape (Apis mellifera L.) per la fruttificazione del melone (Cucumis melo L.) in coltura protesta. Bullettino dell'Istituto di Entomolodia della Università di Bologna, V.36, P,91-114, 1981

COUTO, R.H.N.; COUTO, L.A. Apicultura: Manejo e produtos. Jaboticabal:FUNEP, 1996. 154p.

D'AGUILAR, I. et al. Problèmes de pollinisation des cultures sous abri.

Bull. Tech. Inf. Ingrs Servs agric., n.217, p. 185-188, 1967.

FÁVERO, A.C. et al. Biologia floral e insetos polinizadores em flores de melão (Cucumis melo var reticulatus variedade bônus $\mathrm{n}^{\circ} 2$ ). In: CONGRESSO BRASILEIRO DE APICULTURA, Anais... Campo Grande:CBA, 2002

FREE, J.B. Insect pollination of crops. London: Academic Press, 1993. $684 p$.

GARCIA, R.C. et al. Estudo da polinização do melão (Cucumis melo) por Apis mellifera, em estufa. In: www.unimar.br/ciencias/7-19-1.html. consulta em 03/10/2005 
HOLANDA NETO, J.P. et al. Diferenças na atratividade de oito híbridos de melão (Cucumis melo) para a abelha melífera (Apis mellifera). In: CONGRESSO BRASILEIRO DE APICULTURA, Anais...Florianópolis:CBA, 2000.cd-room INSTITUTO AGRONÔMICO DE CAMPINAS (IAC). Boletim Técnico, 100. Campinas: IAC, 1996

ISELIN, W. A. et al. The pollination of melons in air inflated greenhouses by HoneyBees. Environmental Entomology, College Park, v. 3, n. 4, 664-666, 1974.

KATO, E.C.; COUTO,R.H.N; Polinização em melão (Cucumis melo L.) dos tipos amarelo e rendilhado. Naturalia, v.27, p. 201-210, 2002.

LEMASSON, M. Intérêt de l'abeille mellifère (Apis mellifera) dans la pollinisation de cultures en serre de cornichon (Cucumis sativus), de melon (Cucumis melo) et de tomate (Lycopersicum esculentum). Revue de l'Agriculture, v. 40, p. 915-924, 1987.

McGREGOR, S.E.; TODD, F.E. Cantaloupe production with honeybees. J. econ, Ent., n. 45, p.43-47, 1952.

SAKAMORI, M. et al. Growing muskmelons with honeybees as pollinators. Research Bulletin of the Aichi-ken Agricultural Research Center, B (Horticulture). V.9, p. 15-21, 1977.

TAYLOR, E.A. Cantaloupe production increased with honey bees. J. econ. Ent., v. 48, p. 327, 1955.

WILLIAMS, P. Pollination of melons. Aust. Bee J. , v.68, p. 18-21, 1987 